Agenor Garcia Junior

\title{
Toracometria em crianças com distrofia muscular de Duchenne: refinamento de metodologia de avaliação
}

Dissertação apresentada à Faculdade de Medicina da Universidade de São Paulo para obtenção do título de Mestre em Ciências

Programa de Ciências da Reabilitação

Área de concentração: Movimento, Postura e Acão Humana Orientadora: Profa. Dra. Fátima Aparecida Caromano 
Agenor Garcia Junior

\title{
Toracometria em crianças com distrofia muscular de Duchenne: refinamento de metodologia de avaliação
}

\author{
Dissertação apresentada à Faculdade de Medicina \\ da Universidade de São Paulo para obtenção do \\ título de Mestre em Ciências \\ Programa de Ciências da Reabilitação \\ Área de concentração: Movimento, Postura e Acão Humana \\ Orientadora: Profa. Dra. Fátima Aparecida Caromano
}

(Versão corrigida. Resolução CoPGr 5890, de 20 de dezembro 2010.

A versão original está disponível na Biblioteca FMUSP)

São Paulo

2011 


\section{Dados Internacionais de Catalogação na Publicação (CIP)}

Preparada pela Biblioteca da

Faculdade de Medicina da Universidade de São Paulo

Creprodução autorizada pelo autor

\section{Garcia Junior, Agenor}

Toracometria em crianças com distrofia muscular de Duchenne : refinamento de metodologia de avaliação / Agenor Garcia Junior. -- São Paulo, 2011.

Dissertação(mestrado)--Faculdade de Medicina da Universidade de São Paulo. Programa de Ciências da Reabilitação. Área de concentração: Movimento, Postura e Ação Humana.

Orientadora: Fátima Aparecida Caromano.

Descritores: 1.Modalidades de Fisioterapia 2.Reprodutibilidade dos resultados 3.Avaliação respiratória 4.Distrofia muscular de Duchenne

USP/FM/DBD-271/11 


\section{Dedicatória}

Dedico este trabalho a minha esposa Carla e aos meus filhos Nicole, Murilo e Camile que souberam me estimular nos momentos certos, não me deixaram esmorecer nos momentos de desânimo. Em especial a minha filha Nicole pelas "broncas e puxões de orelha" nos momentos de desalento. 
Agradecimento

Agradeço a todos de que alguma forma, direta e indiretamente me ajudaram a realizar este trabalho.

Aos pacientes que me ajudaram nesta empreitada.

Especialmente a minha orientadora Fatima Aparecida Caromano pela paciente, compreensão, confiança e generosidade, em todos as etapas deste trabalho.

As pessoas que colaboraram diretamente com seus conhecimentos;

Adriana Maria Contesini

Renata Escorcio

Lilian Aparecida Yoshimura Fernandes

Sílvia Maria Amado João

Ao Genoma, Associação Brasileira de Distrofia Muscular (ABIDIM) pela generosidade de permitirem a utilização de suas instalações e o contato com pacientes e alunos. 


\section{Normas de confecção do texto}

Esta dissertação ou tese está de acordo com as seguintes normas, em vigor no momento desta

publicação:

Referências: adaptado de International Committee of Medical Journals Editors (Vancouver).

Universidade de São Paulo. Faculdade de Medicina. Divisão de Biblioteca e Documentação.

Guia de apresentação de dissertações, teses e monografias. Elaborado por Anneliese Carneiro da Cunha,

Maria Julia de A. L. Freddi, Maria F. Crestana, Marinalva de Souza Aragão, Suely Campos

Cardoso,

Valéria Vilhena. 3a ed. São Paulo: Divisão de Biblioteca e Documentação; 2011.

Abreviaturas dos títulos dos periódicos de acordo com List of Journals Indexed in Index Medicus. 

ÍNDICE

Dedicatória

Agradecimentos

Normas de Confecção do texto iii

Lista de Siglas

Resumo

Summary

Introdução

Distrofia muscular de Duchenne - função respiratória 2

Postura Sentada e Função Respiratória 5

$\begin{array}{ll}\text { Toracometria em crianças com distrofia muscular de Duchenne - } & 7\end{array}$

Refinamento de metodologia de avaliação

Materiais e métodos

Procedimentos $\quad 9$

Coleta de dados $\quad 11$

Análise de dados $\quad 11$

$\begin{array}{ll}\text { Resultados } & 13\end{array}$

Análise de confiabilidade $\quad 14$

Discussão $\quad 22$

Conclusões 25

Anexos

Anexo I 26

Anexo II $\quad 29$

Anexo III $\quad 30$

Anexo IV 31

Referências $\quad 32$ 


\section{RESUMO}

Contextualização: A toracometria é uma técnica simples e acessível para avaliar mobilidade do tórax durante respiração forçada, mas não permite analisar os movimentos compensatórios utilizados por portadores de doenças crônicas, como a distrofia muscular de Duchenne (DMD), caracterizada pela degeneração progressiva e irreversível da musculatura esquelética. Objetivos: Ampliar a técnica de toracometria, permitindo avaliação das compensações posturais, analisar a confiabilidade da ferramenta e, descrever a mobilidade torácica de crianças com DMD, durante respiração profunda. Métodos: Participaram 60 meninos, $30 \mathrm{com}$ DMD (10,1 $\pm 0,5$ anos) e 30 saudáveis $(9,5 \pm 0,6$ anos). Aplicou-se a técnica de toracometria expandida em duas fases: livre movimentação corporal, permitindo avaliação de compensações posturais (toracometria livre) e, bloqueio de movimentos compensatórios, facilitando análise de movimentos do gradil costal (toracometria dirigida). Esta técnica prevê filmagem e observação sistemática dos movimentos, gerando dados numéricos e descritivos permanentes. $\mathrm{O}$ estudo de confiabilidade da técnica foi realizado nos dois grupos. Resultados: As medidas de toracometria axial e xifóide (livre e dirigida) apresentaram excelente confiabilidade intra e interexaminadores. As medidas coletadas apresentaram diferenças significantes entre os grupos. Nas crianças com DMD, a toracometria livre apresentou valor de expansibilidade torácica maior, mostrando que as compensações facilitam a mobilidades da caixa torácica. A técnica de toracometria livre apresentada permitiu a descrição de compensações posturais, sendo que as observadas com maior frequência foram os movimentos associados de cabeça, ombro e tórax. Conclusões: A técnica de toracometria expandida apresentou excelente confiabilidade e permitiu descrever os movimentos do tórax e dos arcos costais. Sugere-se sua utilização na avaliação respiratória de crianças com DMD.

Palavras-chave: Fisioterapia, reprodutibilidade de resultados, avaliação respiratória, distrofia muscular de Duchenne 


\section{SUMMARY}

Background: Thoracic cirtometry is a simple and accessible technique to assess chest mobilization during forced breathing, but does not allow to analyze compensatory movements commonly used by people with chronic diseases, such Duchenne muscular dystrophy (DMD), characterized by progressive degeneration of musculoskeletal system. Objectives: To expand the technique of thoracic cirtometry to allow assessment compensatory movements, to analyze the reliability of the tool and to describe thoracic mobility of children with DMD. Methods: Participated 60 boys, 30 with DMD (10.1 \pm 0.5 years) and 30 healthy $(9.5 \pm 0.6$ years $)$. The expanded thoracic cirtometry was organized in two phases: body can move freely, allowing the assessment of postural compensations (free thoracic cirtometry) and with postural movements blocked, facilitating the analysis of movements of the rib cage (conducted thoracic cirtometry). This technique provides systematic observation and recording of body movements, creating descriptive and numeric permanent data. We investigated repeatability and reproducibility. Results: Measures of axial and the xiphoid thoracic cirtometry (free and conducted) showed excellent reliability. All measures showed significant differences between groups. In the DMD boys, free thoracic cirtometry presented a greater value, which probably occurs due to postural compensations. The most commons were movements of head, shoulder and chest. Conclusions: The expanded thoracic cirtometry technique showed excellent reliability and achieved the objectives of determining values related to ribs mobility during deep breath, and to describe compensatory movements. We suggested its use in respiratory evaluation of children with DMD.

Keywords: Physiotherapy, reproducibility of results, respiratory assessment, Duchenne muscular dystrophy 


\section{INTRODUÇÃO}

O presente trabalho teve como motivação a dificuldade de avaliação e monitorização dos portadores de Distrofia Muscular de Duchenne. Os testes de função pulmonar, além de serem custosos, são de difícil acesso para maioria da população. A toracometria consiste num sistema simples e de fácil manejo e execução. A necessidade de verificarmos a confiabilidade e a repetibilidade, nos levou a realizarmos uma divisão em dois grupos um com crianças normais e outro com portadoras de distrofia muscular de Duchenne e dividirmos a toracometria em livre e dirigida, a fim de criarmos possibilidades de análise diferentes.

Os dois grupos foram submetidos a aos dois tipos de avaliação e seus dados comparados

A fundamentação deste estudo passa pela revisão da evolução da disfunção respiratória em pessoas com DMD, e a influência das mudanças de postura (mecanismos de compensação ou simplesmente a mobilidade) do tronco, a partir da posição sentada (escrito em parceria com Adriana M. Contesini). Estes temas serão abordados em capítulos separados.

O experimento será apresentado nesta dissertação, tal qual foi escrito para publicação. 


\section{REVISÃO DE LITERATURA}

\section{Distrofia muscular de Duchenne - função respiratória}

A distrofia muscular do tipo Duchene (DMD), é um distúrbio genético de caráter recessivo com alta taxa de mutação, cuja alteração inicial está localizada no cromossomo Xp21. Manifesta-se no sexo masculino, sendo as mulheres portadoras, desenvolvendo-se excepcionalmente em dois casos, na síndrome de Turner (45X) e nos casos em que os dois genes herdados forem afetados. O distúrbio genético é causador da diminuição da proteína distrofina na superfície dos músculos causando uma ruptura na membrana do sarcômero $(\text { gaps })^{(54,55)}$, levando a desintegrações locais destes e causando uma lesão da fibra muscular esquelética de caráter progressivo e irreversível, podendo acometer a musculatura cardíaca e o sistema nervoso ${ }^{(01,02)}$.

Sua incidência é de um para cada três mil e quinhentos nascidos vivos do sexo masculino. No Brasil são setecentos novos casos de DMD por ano ${ }^{(02)}$. As investigações demonstram que cerca de um terço ou mais dos casos são decorrentes de novas mutações ${ }^{(54)}$.

LEVY (1978) afirmou que as manifestações clínicas normalmente começam nos primeiros três anos de vida e os afetados não são capazes de andar após os dezesseis anos de vida. Um dos achados iniciais da doença é o aumento da musculatura do tríceps sural, acompanhado frequentemente pelo envolvimento funcional inicial dos movimentos do cíngulo do membro inferior. No exame físico encontra-se aumento do volume muscular, sendo que sua palpação demonstra uma firmeza e resistência anormais, achado este caracterizado como pseudo-hipertrofia, que pode acometer outros grupos musculares como os músculos masseter, deltóide e serrátil anterior ${ }^{(55)}$. Inicialmente encontra-se comprometimento simétrico da musculatura, acompanhado de déficit motor dos músculos do cíngulo do membro inferior e, posteriormente, outros grupos musculares como os do cíngulo do membro superior são afetados de maneira progressiva ${ }^{(56)}$. 
Após a restrição ao leito, o paciente apresenta uma progressão rápida da perda de força muscular devido à imobilidade, levando a comprometimento respiratório ${ }^{(13)}$. A degeneração progressiva da musculatura afeta de forma sistêmica o organismo e a musculatura respiratória, gerando alterações na contratibilidade, acarretando que o indivíduo necessita gerar compensações biomecânicas a fim de manter sua capacidade vital dentro de uma faixa de normalidade, que possibilite a este manter a melhor oxigenação possível dos tecidos. Dentre as alterações podemos encontrar as relacionadas à caixa torácica, como a escoliose, cifose, lordose, abaulamento, elevações de últimos arcos costais e as de caráter muscular como a solicitação da musculatura acessória, que consiste nos musculos esternocleidomastóideo, peitoral maior e trapézio. No caso de uma situação em que seja necessária uma inspiração forçada os músculos posturais podem ser acionados. Estudos realizados revelam que a redução da capacidade vital é maior quanto mais severa for a escoliose, mostrado que o aumento de dez graus da curvatura implica em diminuição de quatro por cento da capacidade vital $^{(26)}$.

A maior causa de óbito destes indivíduos é a falha respiratória ou infecção pulmonar, ocorrendo em torno $75 \%$ dos $\operatorname{casos}^{(57)}$.

A capacidade vital começa a decair logo aos sete anos de idade, sendo que aos quatorze anos atinge metade do valor esperado e, aos vinte e um atinge vinte por cento do esperado. O padrão respiratório destes pacientes com fraqueza muscular é de respiração rápida e superficial, com volume corrente menor do que em pessoas sadias, e está associado com a presença de alterações nas pressões parciais dos gases sanguíneos. A complacência encontra-se reduzida. Os músculos intercostais internos e abdominais, que auxiliam a expiração, também são acometidos, reduzindo a efetividade da tosse e conseqüentemente a eliminação de secreções ${ }^{(57,58)}$.

Observa-se diminuição gradual da capacidade vital na adolescência, comprometendo também os volumes de reserva inspiratório, aumentando o volume residual, correlacionados à fraqueza muscular progressiva e a escoliose ${ }^{(22,23,24,25)}$. Quando a curvatura da coluna excede $35^{\circ}$ nestes indivíduos, a capacidade vital encontra-se menor que $40 \%$ dos valores normais ${ }^{(26)}$.

Durante o sono, o déficit muscular se agrava, principalmente em decúbito dorsal ou ventral, onde a musculatura respiratória encontra-se em desvantagem biomecânica, acarretando quadros de hipopnéia e apnéia que produzem fragmentação do sono e sonolência diurna, às vezes acompanhada 
de cefaléia matinal, fadiga, baixa concentração e alterações psicológicas, incluindo confusão e depressão ${ }^{(57)}$.

$\mathrm{Na}$ avaliação do tórax, utilizando o exame físico para fornecer o diagnóstico fisioterapêutico, criando condições para o início da terapêutica preventiva ou curativa e estabelecer o provável prognóstico do paciente. Na determinação das disfunções torácicas é necessário um exame específico que inclui a avaliação da mobilidade torácica por meio da cirtometria $^{11}$.

Este método tem despertado bastante interesse, por se tratar de uma técnica acessível e de fácil execução, que fornece dados quantitativos da mobilidade torácica.

A toracometria consiste na realização de medidas das circunferências do tórax gerando informações sobre os movimentos respiratórios. ${ }^{16}$ Sendo uma forma simples e acessível de avaliar a expansibilidade torácica, apenas uma fita métrica é necessária. Encontraram-se, na literatura, uma série de propriedades atribuídas à cirtometria, diversos protocolos que adotaram diferentes posturas e pontos anatômicos de referência. ${ }^{16-}$ $18,20,21$ 


\section{Postura Sentada e Função Respiratória}

A adoção da postura bípede pelo homem trouxe mudanças aos diferentes sistemas corporais $^{(1)}$. A respiração é uma função primordial para a sobrevivência do indivíduo e seu sistema constitui-se de uma interação entre meio interno e externo ${ }^{(2,6)}$. Com as mudanças estruturais ocorridas no decorrer da evolução humana, o sistema respiratório sofreu alterações das relações entre coluna vertebral e crânio, alterações da orofaringe, ligeira redução da altura da caixa torácica e deslocamento das costelas, dando ao tórax uma forma mais aplanada para permitir uma melhor adaptação à força da gravidade na posição ortostática. Os gradientes circulatórios e ventilatórios dos pulmões passaram de um eixo dorsoventral para um ápico-basal, enquanto os músculos respiratórios modificaram sua disposição estrutural e funcional ${ }^{(1)}$.

A caixa torácica apresenta íntima relação com os pulmões, o ciclo respiratório exige sincronismo entre a caixa torácica, o pulmão e a musculatura, atuando de forma $\operatorname{coordenada}^{(7)}$. Inúmeros fatores podem influenciar seu funcionamento, tais como fatores hormonais, emocionais, de comportamento, postura, medicamentos, entre outros ${ }^{(2,6)}$. Sabe-se que, mesmo tarefas passivas como realização de cálculos mentais, exposição a ruídos ou a imagens aversivas podem causar alterações na resistência das vias aéreas, uma vez que as respostas respiratórias são dependentes da estimulação autonômica, causando alterações dos parâmetros ventilatórios do indivíduo ${ }^{(6)}$.

Além disso, alterações no posicionamento corporal e na atuação das forças da gravidade, entre outros fatores, ocasionam mudanças na função respiratória, em diferentes intensidades e associada a diferentes patologias ${ }^{(1)}$.

Os efeitos da postura sobre a função respiratória têm motivado inúmeros pesquisadores que analisaram dados referentes à função pulmonar com o objetivo de rastrear todas as alterações envolvidas nos mecanismos de adaptação à mudança da postura corporal por meio de diferentes recursos $^{(6,8,15)}$. Para o fisioterapeuta a importância da organização e divulgação deste conhecimento está na necessidade de compreender e fundamentar as orientações a serem fornecidas para pessoas que permanecem grande parte do dia na postura sentada, além de permitir o entendimento de como estas alterações podem interferir na função respiratória de indivíduos saudáveis e inferir, a partir daí, sua 
importância para pessoais em condições especiais, como obesos, gestantes e usuários de cadeira de rodas, entre outros.

Os efeitos da postura sobre a função respiratória têm motivado pesquisas com o objetivo de rastrear alterações nos mecanismos de adaptação à mudança da postura corporal.

Nos estudos sobre postura sentada observa-se que as alterações são significativas em indivíduos com doenças pulmonares, cardíacas e nos idosos, entre outros e que, mesmo em indivíduos saudáveis as alterações nos testes de função podem ultrapassar a variação dos valores considerados normais para uma dada posição. A revisão do tema mostrou que faz-se necessários estudos para determinar o efeito cumulativo destas alterações que podem ser significativas em indivíduos saudáveis, com algum fator complicador, como por exemplo, a obesidade ou a gravidez, e quais as alternativas possíveis para minimizar estes efeitos. 


\section{Toracometria em crianças com distrofia muscular de Duchenne - expansão de metodologia de avaliação}

Distrofia Muscular de Duchenne (DMD) é a segunda desordem genética mais comum em humanos. Constituí um distúrbio genético de caráter recessivo, com alta taxa de mutação, localizado em Xp21. Esta mutação causa um defeito na estrutura da membrana muscular, que tem como consequência a ausência ou diminuição da produção da proteína distrofina, responsável, entre outras funções, pela integridade da membrana basal da fibra muscular. O quadro clínico da DMD caracterizase principalmente pela degeneração progressiva e irreversível da musculatura esquelética, levando incapacidade de deambulação e insuficiência respiratória por fraqueza muscular. ${ }^{(1)}$

Em decorrência da fraqueza da musculatura postural e respiratória, a maioria dos pacientes com DMD desenvolvem escoliose e/ou deformidades na caixa torácica, com comprometimento da biomecânica respiratória $^{(2,3)}$, e diminuição dos volumes pulmonares, incapacitando a expansão até sua capacidade máxima e o retorno ao volume resídual ${ }^{(4,5)}$, resultando em uma ventilação inadequada em situação de aumento das cargas mecânicas e necessidades elevadas de ventilação $^{(6)}$.

Uma ação abrangente, multi e interdisciplinar, organizada para manter o paciente em sua melhor condição física possível é a melhor opção ${ }^{(1)}$, pois, tem uma função preventiva e atua nos aspectos primários e secundários da doença, podendo favorecer a história natural e melhorar a qualidade de vida ${ }^{(7)}$. A preocupação com a função respiratória se justifica por ser a falência respiratória a principal causa de morte nesta população.

Nas avaliações fisioterapêuticas de pacientes com patologias respiratórias, a mensuração da amplitude de movimento torácico, por meio da toracometria, também denominada perimetria torácica, tem sido utilizada devido a sua praticidade, rapidez e baixo custo de execução ${ }^{(8)}$.

Em situações de inspiração forçada, na presença de fraqueza muscular, os músculos posturais podem ser acionados, gerando compensações biomecânicas. ${ }^{(3)}$ Estas compensações, embora positivas em casos de pacientes com doenças degenerativas progressivas, , por fornecerem uma estratégia de 
compensação para obtenção da ação desejada,são pouco estudadas e desconsideradas durante a avaliação da mobilidade torácica. A compreensão do movimento do gradil costal à custa da musculatura respiratória e dos movimentos compensatórios, decorrentes do uso de musculatura acessória e postural são informações complementares que auxiliam na compreensão do quadro da mobilidade torácica associada com a respiração profunda. Conhecer as possibilidades de movimentos compensatórios que podem auxiliar os pacientes com DMD em situações onde sejam exigidas respirações forçadas, pode auxiliar o fisioterapeuta a estabelecer, junto com o paciente, estratégias com comportamentos motores previamente treinados.

Diante do exposto, os objetivos deste estudo foram expandir a técnica de coleta de dados de toracometria, de tal forma a permitir a avaliação das compensações posturais, analisar a confiabilidade intra e interexaminador da ferramenta quando aplicada em crianças saudáveis e portadoras de DMD e, caracterizar os movimentos torácicos destas durante a respiração profunda. 


\section{Método}

Estudo aprovado pelo Comitê de Ética da Faculdade de Medicina da Universidade de São Paulo, no processo número 0685/09.

\subsection{Sujeitos:}

Participaram 60 crianças do sexo masculino, sendo 30 com distrofia muscular de Duchenne (10,1 $\pm 0,5$ anos $)$ - grupo DMD e, 30 saudáveis $(9,5 \pm 0,6$ anos $)$ - grupo $\mathrm{S}$.

O critério de inclusão foi á ausência de doença respiratória crônica.

Critérios de exclusão do estudo, tanto para o grupo DMD quanto para o grupo S, a presença de qualquer doença aguda como lesões musculoesqueléticas, gripes e resfriados durante o período de coleta de dados e a incapacidade de compreensão do teste proposto.

O estudo foi realizado no Laboratório de Fisioterapia e Comportamento do Curso de Fisioterapia da FMUSP, na Associação Brasileira de Distrofia Muscular (ABDIM) e, em uma Escola Estadual de Ensino Fundamental da Cidade de São Paulo.

\subsection{Procedimentos:}

\section{Expansão do método de toracometria}

A medida da amplitude torácica, como descrita na literatura, é realizada por meio da cirtometria, utilizando-se uma fita métrica, escalonada em centímetros $(\mathrm{cm})$, e coletada em duas regiões, a saber, na altura das axilas e na altura do processo xifóide. As referências usadas para as coletas de medidas são, na altura das axilas, no plano frontal, o terceiro espaço intercostal. No plano dorsal, é utilizado o processo espinhoso da quinta vértebra torácica. Para coleta de medida na altura do processo xifóide, no plano frontal, é usada a ponta do apêndice xifóide, e no plano dorsal o processo espinhoso da décima vértebra torácica ${ }^{9}$.

Em trabalhos recentes usando homens adultos jovens, a coleta da toracometria, como utilizada tradicionalmente, mostrou ser uma técnica replicável e utilizável na prática clínica ${ }^{9,10}$.

Sugerimos manter a coleta destas medidas e complementar os dados, coletando as mesmas medidas permitindo-se ao paciente, a realização de todos os movimentos de cabeça, tronco e membros superiores que se façam necessários para que este realize inspiração e expiração profundas, permitindo assim, a visualização dos movimentos compensatórios. 
Propomos que a coleta de dados com movimentos livres, que denominamos toracometria livre, seja precedida da coleta com os movimentos bloqueados (tradicional), que chamamos de toracometria dirigida. Para o estudo dos movimentos compensatórios, propomos que a coleta de dados da toracometria, seja filmada e observada de forma sistemática, isto é, de tal forma que, cada seguimento corporal (cabeça, pescoço, membros superiores e tronco) possa ser observado quantas vezes se faça necessário até que o examinador conclua sobre a presença, ou não de movimentos compensatórios, e descreva o tipo de movimento executado. O dado deve ser registrado para posterior análise.

Para coletar os dados utilizando a técnica de toracometria expandida, orienta-se o voluntário para que realize uma inspiração máxima e logo após uma expiração máxima, enquanto que a fita métrica, colocada ao redor do tórax, em posições anatômicas definidas, desloca-se acompanhando a expansão do tórax. O procedimento é aplicado em nível superior e inferior do tórax e o examinador acompanha os movimentos dos pacientes. Para cada variável devem ser coletadas 3 medidas relativas à inspiração e à expiração forçadas e a diferença entre ambas é registrada, ou seja, a medida coletada diz respeito à medida da circunferência torácica no pico de inspiração menos a medida coletada no pico de expiração.

Para fins de pesquisa, consideramos a melhor medida obtida. Para evitar cansaço permitimos um descanso mínimo de um minuto entre cada coleta.

O comando verbal deve ser claro e reforçador. Tradicionalmente, pede-se ao paciente para não deslocar o tronco ou a cabeça para frente ou para trás, nem movimentar os braços. Esta ação impede movimentos compensatórios, permitindo a avaliação isolada da mobilidade da caixa torácica. Durante a cirtometria livre, que deve anteceder a cirtometria induzida, não é fornecida nenhuma informação sobre postura ou movimentos.

\section{Coleta de dados}

Um examinador previamente treinado durante 8 horas (quando avaliou 10 crianças saudáveis e 10 com DMD), coletou as medidas de toracometria do grupo S e DMD, no tempo zero e um mês após a primeira coleta, gerou os dados para análise de confiabilidade intraexaminador. Dois dias após a coleta do tempo zero, outro examinador também previamente treinado coletou as mesmas medidas, gerando dados para análise de confiabilidade interexaminadores. 
Os testes foram filmados com a filmadora colocada perpendicularmente num ângulo de $45^{\circ}$ ao sujeito, sobre um tripé a um metro de altura, numa distância de 2 metros. $\mathrm{O}$ examinador se posicionou no lado oposto da filmadora, permitindo visualização da criança em avaliação.

A partir dos filmes, foram realizadas observações sistemáticas para registrar os movimentos compensatórios realizados pelas crianças durante a inspiração e expiração forçadas.

A coleta de dados e a observação dos filmes foram executadas por avaliadores (pesquisador e dois avaliadores externos) que também se submeteram a treinamento de 8 horas.

\section{Análise dos dados}

Para caracterização dos sujeitos e estudo das variáveis de compensações foram realizadas análises descritivas.

Por se tratar de variáveis quantitativas em escala de razão (toracometria) e compensações observadas, foi testada a normalidade de cada variável por meio do teste de aderência - KolmogorovSmirnov. Verificada a normalidade, foram realizados os testes de Barlett e Levene para verificar a homogeneidade das variáveis ${ }^{(11,12)}$.

Confirmado os pressupostos, foram realizadas as correlações das toracometria livre e dirigida para as medidas axial e xifóide, por meio do teste de correlação de Pearson. Foram feitas as comparações entre toracometria livre e dirigida entre os grupos (grupos S e DMD) por meio da análise de variância (ANOVA) two-way, tendo como fatores os grupos e as medidas de toracometria.

Foi pesquisado o índice de correlação intra-classe (ICC), utilizando-se os resultados da ANOVA two-way para a reprodutibilidade dos dados e ANOVA one-way com medidas repetidas para cálculo da repetibilidade dos dados avaliados, para que deste modo se pudesse refletir tanto o grau de correspondência quanto à concordância entre os avaliadores. ${ }^{13,14}$

Para avaliar o nível de confiança entre os diferentes examinadores (reprodutibilidade), a amostra foi avaliada por dois examinadores diferentes, treinados, sem conhecimentos entre eles dos resultados obtidos. Para analisar o nível de confiança de um mesmo examinador (repetibilidade), os mesmos sujeitos foram examinados em duas ocasiões diferentes com um intervalo de um mês, para que este examinador não memorizasse os resultados. ${ }^{(15,16} 4$. Resultados 


\subsection{Caracterização dos participantes}

As informações sobre peso, altura e idade estão descritas na Tabela 1.

Tabela 1 - Características dos indivíduos estudados quanto à idade,_peso e altura, e o resultado da análise de diferença entre grupos entre os grupos DMD e S (teste t).

\begin{tabular}{lcccc}
\hline & $\begin{array}{c}\text { DMD }(\mathbf{n}=\mathbf{3 0}) \\
\text { (cadeirantes } \mathbf{p})\end{array}$ & $\mathbf{S}(\mathbf{n}=\mathbf{3 0})$ & valor de $\mathbf{p}$ & TEST t \\
& $10,1 \pm 0,5$ & $9,5 \pm 0,6$ & 0,332 & \\
Idade (anos) & $41,70 \pm 1,20$ & $37,40 \pm 1,34$ & 0,009 & $*$ \\
Peso (kg) & $131 \pm 2,3$ & $139 \pm 3,6$ & 0,003 & $*$ \\
\hline
\end{tabular}

Valores expressos em Média \pm desvio padrão. *Diferença significativa $(\mathrm{p}<0,05)$.

Foram organizados dois grupos, com idade próxima (DMD e S) e encontrou-se diferença significativa no peso e na altura, como esperado, uma vez que a DMD afeta o ganho de peso e o desenvolvimento destas crianças. As crianças com DMD se mostraram mais baixas e mais pesadas. $\mathrm{O}$ ganho de peso nesta população pode estar associado à inatividade. ${ }^{7}$

$\mathrm{Na}$ avaliação física dos indivíduos, realizada por observação direta e executada por fisioterapeuta experiente, detectou-se no grupo DMD, 97\% das crianças apresentam algum tipo de deformidades no tórax, sendo as mais evidentes o abaulamento à direita (46\%) e o tórax em tonel (23\%). Neste grupo, 57\% das crianças apresentaram deformidades anatômicas de tórax relacionadas com a coluna vertebral sendo que, destas, $46 \%$ 
apresentavam escoliose à direita, $10 \%$ à esquerda e $1 \%$ com escoliose em S. Estas alterações dificultaram, mas não impediram a coleta de dados.

No grupo S não foi encontrada deformidade de tórax.

\subsection{Análise de confiabilidade}

Foi pesquisada a confiabilidade interavaliador (reprodutibilidade) e intra-avaliador (repetibilidade) da toracometria (axial e xifóidea), e a observação seguida de registro de presença de compensações de movimentos respiratórios forçados das crianças dos grupos DMD e S. Conforme mostrado na Tabela 2 (no final deste item), todas as medidas pesquisadas, para ambos os grupos, apresentaram uma excelente confiabilidade, demonstrando precisão da coletas de dados para avaliação da expansibilidade torácica nas condições propostas.

Foram realizadas as medidas de toracometria livre e dirigida em crianças com DMD e em crianças saudáveis e comparadas. Todas as medidas de toracometria apresentaram-se diferentes significativamente entre os grupos $\mathrm{S}$ e DMD, dados os respectivos valores de $p$, conforme mostrado na Tabela 3(no final deste item). O mesmo pode ser afirmado em relação ao número de compensações posturais realizadas, considerando-se cada segmento corporal isoladamente.

Os valores da toracometria das crianças portadoras de Duchenne apresentam uma diferença estatística entre os valores da medida axial livre e dirigida, sendo que a livre apresenta um valor de expansibilidade torácica maior, conforme mostrado na Tabela 4(no final deste item). 
Tabela 2 - Análise de repetibilidade (intra-examinador) e e reprodutibilidade (interexaminador) das medidas de toracometria e compensações nos grupos DMD e S

Análise de repetibilidade - Grupo DMD

\begin{tabular}{|c|c|c|c|c|c|}
\hline \multirow[t]{2}{*}{ Toracometria } & & $1^{\mathrm{o}}$ & $2^{\circ}$ & \multirow[b]{2}{*}{ ICC } & \multirow[b]{2}{*}{ Classificação } \\
\hline & Tipo & Avaliação & Avaliação & & \\
\hline \multirow[t]{2}{*}{ Axial } & & & $2,5 \pm 0,9$ & & \\
\hline & Livre & $2,6 \pm 1,0$ & & 0,90 & Excelente \\
\hline \multirow{2}{*}{ Axial } & & & $1,8 \pm 0,9$ & & \\
\hline & Dirigida & $1,8 \pm 0,8$ & & 0,96 & Excelente \\
\hline \multirow[t]{3}{*}{ Xifóide } & & & $2,2 \pm 0,9$ & & \\
\hline & & $2,1 \pm 1,1$ & & 0,95 & Excelente \\
\hline & Livre & & & & \\
\hline \multirow[t]{2}{*}{ Xifóide } & & & $1,6 \pm 0,8$ & & \\
\hline & Dirigida & $1,7 \pm 0,9$ & & 0,90 & Excelente \\
\hline \multicolumn{6}{|l|}{ Compensações } \\
\hline por segmento & $3,8 \pm 0,5$ & $3,8 \pm 0,4$ & 0,93 & 0,93 & Excelente \\
\hline
\end{tabular}


Análise de repetibilidade - Grupo S

\begin{tabular}{cccccc}
\hline Toracometria & Tipo & $1^{\mathbf{0}}$ & $2^{\mathbf{0}}$ & ICC & Classificação \\
& & Avaliação & Avaliação & & \\
Axial & Livre & $5,1 \pm 0,9$ & $5,1 \pm 0,8$ & 0,99 & Excelente \\
Axial & Dirigida & $3,8 \pm 0,9$ & $3,8 \pm 0,9$ & 1,00 & Excelente \\
Xifóide & Livre & $4,7 \pm 0,9$ & $4,6 \pm 0,8$ & 0,98 & Excelente \\
Xifóide & Dirigida & $3,6 \pm 0,8$ & $3,6 \pm 0,8$ & & Excelente \\
Compensações & & & & & \\
por segmento & $1,2 \pm 0,5$ & $1,3 \pm 0,2$ & 0,96 & & \\
\end{tabular}


Análise de reprodutibilidade - Grupo DMD

\begin{tabular}{cccccc}
\hline Toracometria & Avaliador & & & & \\
& 1 & Avaliador 2 & ICC & Classificação & P-valor \\
Axial Livre & & & & & \\
& $2,6 \pm 1,0$ & $2,4 \pm 0,9$ & 0,90 & Excelente & 0,757 \\
Axial Dirigida & $1,8 \pm 0,8$ & $2,0 \pm 0,4$ & 0,91 & Excelente & 0,566 \\
& & & & & \\
Xifóide Livre & $2,1 \pm 1,1$ & $2,2 \pm 0,8$ & 0,90 & Excelente & 0,659 \\
Xifóide Dirigida & $1,7 \pm 0,9$ & $1,9 \pm 0,8$ & 0,90 & Excelente & 0,622 \\
& & & & & \\
Compensações por & $3,8 \pm 0,5$ & $3,7 \pm 0,8$ & 0,90 & Excelente & 0,793 \\
segmento & & & & & \\
\hline
\end{tabular}


Tabela 2 - continuidade

Análise de reprodutibilidade - Grupo S

\begin{tabular}{cccccc}
\hline Toracometria & Avaliador & Avaliador 2 & ICC & Classificação & P-valor \\
& 1 & & & & \\
Axial Livre & $5,2 \pm 0,8$ & $5,2 \pm 0,8$ & 1,00 & Excelente & 0,841 \\
Axial Dirigida & $4,5 \pm 0,7$ & $4,6 \pm 0,8$ & 0,98 & Excelente & 0,568 \\
& & & & & \\
Xifóide Livre & $4,6 \pm 0,8$ & $4,6 \pm 0,8$ & 1,00 & Excelente & 0,777 \\
Xifóide Dirigida & $4,3 \pm 0,8$ & $4,5 \pm 0,8$ & 0,97 & Excelente & 0,849 \\
& & & & & \\
Compensações & $1,2 \pm 0,5$ & $1,2 \pm 0,9$ & 0,94 & Excelente & 0,901 \\
por segmento & & & & & \\
\hline$P<0,05$ diferença estatística entre as variáveis. & & & \\
\end{tabular}


Tabela 3 - Tabela demonstrativa da comparação entre as medidas de toracometrias de crianças normais e portadoras de distrofia muscular de Duchenne.

\begin{tabular}{ccccc}
\hline Toracometria & Duchenne & Normal & $F$ & P-valor \\
Axial Livre $(\mathrm{cm})$ & $2,6 \pm 1,0$ & $5,7 \pm 1,6$ & 1,4 & $<0,001^{*}$ \\
Xifóide Livre $(\mathrm{cm})$ & $2,1 \pm 1,1$ & $5,4 \pm 1,6$ & & $<0,001^{*}$ \\
Axial Dirigida (cm) & $1,8 \pm 0,8$ & $4,5 \pm 1,1$ & 0,04 & $<0,001^{*}$ \\
& & & & \\
Xifóide Dirigida (cm) & $1,7 \pm 0,9$ & $4,4 \pm 1,5$ & & $<0,001^{*}$ \\
& & & & \\
\hline$P<0,05$ diferença estatística entre as variáveis. & & & \\
\hline
\end{tabular}

$P<0,05$ diferença estatística entre as variáveis. 
Tabela 4 - Tabela demonstrativa da repetibilidade das medidas de tórax (intra-examinador) Crianças com DMD

\begin{tabular}{ccccc}
\hline Toracometria & Tipo & 1 Avaliação & 2 Avaliação & P-valor \\
Axial & Livre & $2,6 \pm 1,0$ & $2,5 \pm 0,9$ & $<0,001^{*}$ \\
Axial & Dirigida & $1,8 \pm 0,8$ & $1,8 \pm 0,9$ & $<0,001^{*}$ \\
Xifóide & Livre & $2,1 \pm 1,1$ & $2,2 \pm 0.9$ & $<0,001^{*}$ \\
& & & \\
Xifóide & Dirigida & $3,8 \pm 0,5$ & $3,8 \pm 0,4$ & $<0,001^{*}$ \\
& & & \\
\hline$P<0,05$ diferença estatística entre as variáveis. & & \\
\hline
\end{tabular}

$P<0,05$ diferença estatística entre as variáveis. 


\subsection{Movimentos compensatórios (descrição qualitativa)}

A comparação entre o número de compensações posturais apresentadas pelas crianças foi significativamente maior no grupo DMD, com $\mathrm{p}<0,001$.

Durante a toracometria livre foi possível observar que, no grupo DMD, 13 participantes realizaram compensações de ombro com movimentos de elevação e rotação lateral seguida de depressão e rotação medial; extensão seguida de flexão da cabeça; anteriorização com extensão do tronco seguida de retorno à posição inicial, sendo que, 5 participantes utilizaram inclusive, um leve suporte das mãos como alavanca para deslocamento de tronco . Outros 17 participantes utilizaram movimentos compensatórios menos impactantes associando movimentos anteriorização com extensão seguida de retorno à posição inicial do tronco associados com flexão e extensão de cabeça.

No grupo S, 12 participantes realizaram compensação com movimentos de flexão e extensão de cabeça, 4 crianças realizaram anteriorização e retorno à posição inicial do tronco e 8 meninos realizaram compensações com elevação e depressão de ombros, 6 participantes realizaram movimentos associados de elevação e depressão de ombros com flexão e extensão de cabeça. Todas as crianças do grupo $\mathrm{S}$ realizaram compensações, mas com movimentos mais suaves e de menor amplitude, e com número menor de estruturas mobilizadas que as crianças do grupo DMD. 


\section{Discussão}

$\mathrm{Na}$ avaliação e durante as intervenções fisioterapêuticas em diferentes patologias respiratórias, a mensuração da amplitude de movimento torácico tem sido utilizada por ser um método barato, fácil de aprender e aplicar, apropriado para clínica. Mesmo quando o pulmão não está diretamente envolvido, como por exemplo, nas cirurgias de laparotomia, as alterações pulmonares podem ocorrer e a técnica de toracometria pode ser utilizada para gerar indicadores dos bloqueios da mobilidade torácica decorrentes da intervenção ${ }^{17,18,19 .}$ A toracometria, mesmo sendo uma técnica limitada, quando usada de forma criteriosa pode fornecer ao fisioterapeuta dados do estado da funcionalidade da caixa torácica ${ }^{19,20}$.

A avaliação respiratória de crianças portadoras de Duchenne gera dados fundamentais na tomada de decisão clínica, especialmente na rotina do fisioterapeuta, que pode se beneficiar do exame de toracometria.

Um estudo com pacientes asmáticos submetidos à manipulação osteopática demonstrou a eficácia do tratamento, por meio da avaliação por toracometria ${ }^{20,21}$. Em crianças com fibrose cística submetidas a fisioterapia ${ }^{22}$, o estado funcional pulmonar esteve indiretamente refletido na expansibilidade torácica que foi obtida por toracometria.

Em pessoas saudáveis, a eficácia e reprodutibilidade da avaliação da expansibilidade torácica por toracometria, utilizando fita métrica, mostrou ser um método altamente reprodutível e com boa confiabilidade $^{10}$. No presente estudo, todas as medidas de toracometria em ambas as análises (reprodutibilidade e repetibilidade) apresentaram excelente confiabilidade, demonstrando uma precisão dessa medida para avaliação da expansibilidade torácica também em crianças, inclusive nas crianças com DMD. Estes achados facilitam a compreensão de resultados semelhantes quando avaliamos crianças, mesmo estas tendo deformidades torácicas.

Juntamente com a fraqueza simultânea progressiva dos músculos respiratórios ocorre uma insuficiência pulmonar restritiva por alteração postural, alterando a expansibilidade pulmonar. A maioria dos pacientes com DMD desenvolvem uma escoliose progressiva quando eles deixam de deambular e passam a fazer uso da cadeira de rodas. Nesta fase, também desenvolvem ou acentuam as deformidades torácicas e pélvicas ${ }^{23,24}$. Na caracterização e avaliação postural dos nossos participantes 
encontramos alterações posturais sendo que $46 \%$ apresentavam escoliose com concavidade à direita, $10 \%$ a esquerda e $1 \%$ em "S".

Em estudo longitudinal ${ }^{25}$ que avaliou, por observação direta a postura, a correlação entre deformidades torácicas e alterações pulmonares, a diminuição da capacidade vital esteve ligada com piora da deformidade torácica. As deformidades e sua associação com a fraqueza muscular, na DMD, devem ser pesquisadas e acompanhada durante evolução da doença ${ }^{24}$.

Estas alterações progressivas posturais e motoras podem levar essas crianças a construírem estratégias adaptativas para alcançarem o movimento desejado. Em um estudo para avaliar função bimanual em crianças com Duchenne ${ }^{26}$, os autores encontraram que, em consequência da fraqueza muscular característica da doença, essas crianças encontram ajustes antecipatórios posturais para completarem suas funções.

A fraqueza da musculatura respiratória altera os volumes pulmonares, dificultando a expansão pulmonar até sua capacidade total ${ }^{25}$. Além da dificuldade para gerar volume pulmonar, esta perda, associada à fraqueza muscular, também leva à uma dificuldade para gerar mecanismos de defesa, como a tosse $e^{27,28}$. As alterações progressivas respiratórias também podem levar essas crianças a gerar adaptações ventilatórias e a ajustarem a cinemática respiratória com o agravamento da doença, como adotar predominantemente um padrão respiratório abdominal ${ }^{29,30}$.

O presente estudo propôs uma expansão na execução da técnica de toracometria, utilizando-a de duas formas, a toracometria livre e a dirigida, que poderiam fornecer indicadores das compensações posturais facilitadoras da respiração nas crianças portadoras de DMD. Foram encontradas diferenças significativas em todas as medidas de toracometria entre os grupos de crianças saudáveis e com DMD, sendo este último grupo caracterizado por medidas menores. Quando analisados os valores de expansibilidade torácica somente no grupo DMD, e encontramos um valor maior quando foram permitidas compensações na toracometria livre em relação à toracometria dirigida.

Para alcançar maior expansibilidade torácica, essas crianças realizaram, na toracometria livre, compensações como movimentação de cabeça associada ao ombro e estas associadas à movimentação de torácica e lombar. Portanto, assim como para funções motoras, essas crianças criam estratégias compensatórias também para melhora da função respiratória, sendo benéficas, visto que assim elas são capazes de aumentar sua expansibilidade torácica. 
O limite deste trabalho está principalmente na impossibilidade de graduar as compensações realizadas durante a cirtometria livre. Propõe-se o uso de marcadores nas regiões de saliências / proeminências ósseas (ou pontos anatômicos pré-definidos) a fim de se obter referencias que possam gerar dados de angulação dos deslocamentos, em na análise cinemática, permitindo gerar dados mensuráveis, comparáveis e permanentes, uma vez que as imagens geram um banco de dados com filmagens. 


\section{Conclusões}

O método de avaliação testado - toracometria expandida, livre e dirigida, com observação de movimentos compensatórios - apresentou replicabilidade e reprodutibilidade excelentes.

Encontrou-se diferença significativa nas medidas obtidas por meio das duas rotinas de mensuração propostas, que geraram informações complementares na avaliação respiratória destes pacientes.

Foi possível observar que a realização de movimentos compensatórios é comum durante a inspiração seguida de expiração forçadas, e estas compensações envolvem um número maior de seguimentos no grupo DMD.

Sugere-se a utilização do método de toracometria livre e dirigida em rotinas de avaliação pulmonar de crianças com distrofia muscular de Duchenne, ou doenças similares, a exemplo da distrofia muscular de Becker. 
ANEXO I

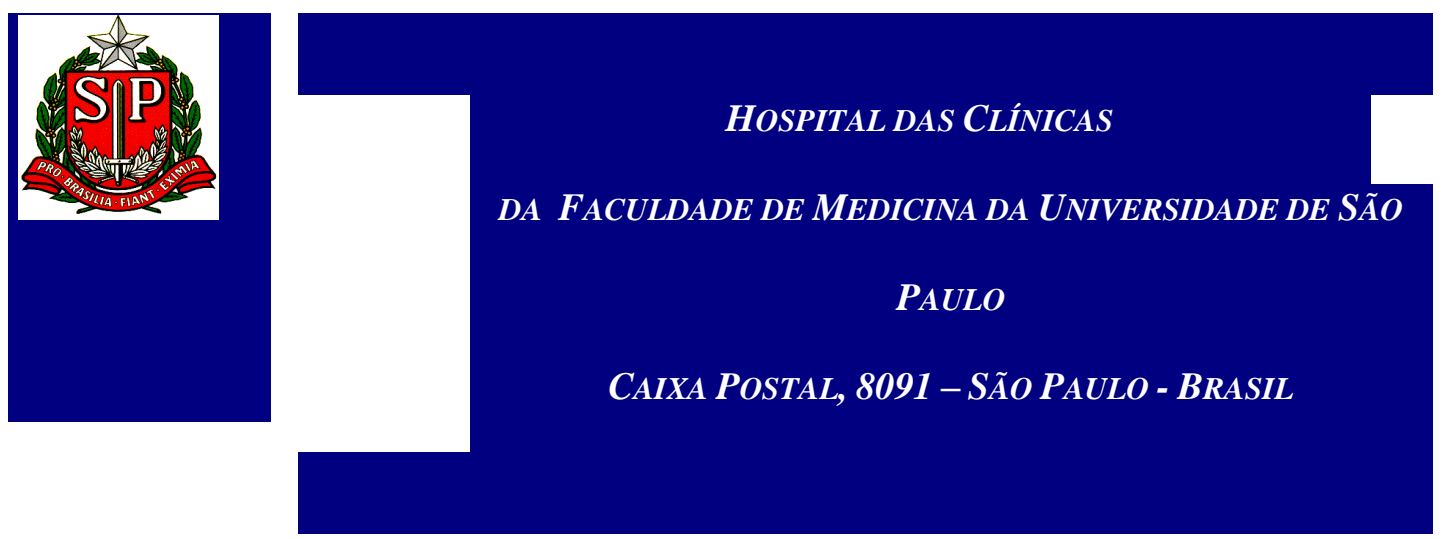

TERMO DE CONSENTIMENTO LIVRE E ESCLARECIDO

(Instruções para preenchimento no verso)

\section{I - DADOS DE IDENTIFICAÇÃO DO SUJEITO DA PESQUISA OU RESPONSÁVEL LEGAL}

1. NOME DO PACIENTE

DOCUMENTO DE IDENTIDADE $\mathrm{N}^{\circ}$ :

SEXO : .M Ž F Ž

DATA NASCIMENTO

$\mathrm{N}^{\circ}$ APTO:

ENDEREÇO CIDADE

BAIRRO

CEP:.

TELEFONE: DDD (

2.RESPONSÁVEL LEGAL

NATUREZA (grau de parentesco, tutor, curador etc.)

DOCUMENTO DE IDENTIDADE : ..SEXO: M Ž F F̌

DATA NASCIMENTO.: .......................

ENDEREÇO: $\mathrm{N}^{\circ}$ APTO:

BAIRRO CIDADE

CEP: TELEFONE: DDD

II - DADOS SOBRE A PESQUISA CIENTÍFICA

1. TÍTULO DO PROTOCOLO DE PESQUISA 
PESQUISADOR:

CARGO/FUNÇÃO:

INSCRIÇÃO CONSELHO REGIONAL N ${ }^{\circ}$

UNIDADE DO HCFMUSP:

3. AVALIAÇÃO DO RISCO DA PESQUISA: SEM RISCO

(probabilidade de que o indivíduo sofra algum dano como consequência imediata ou tardia do estudo)

4.DURAÇÃO DA PESQUISA :

\section{III - REGISTRO DAS EXPLICAÇÕES DO PESQUISADOR AO PACIENTE OU SEU}

REPRESENTANTE LEGAL SOBRE A PESQUISA CONSIGNANDO:

1. justificativa e os objetivos da pesquisa

2. procedimentos que serão utilizados e propósitos, incluindo a identificação dos procedimentos que são experimentais

3. desconfortos e riscos esperados

4. benefícios que poderão ser obtidos

5. procedimentos alternativos que possam ser vantajosos para o indivíduo

\section{IV - ESCLARECIMENTOS DADOS PELO PESQUISADOR SOBRE GARANTIAS DO SUJEITO}

\section{DA PESQUISA CONSIGNANDO:}

1. acesso, a qualquer tempo, às informações sobre procedimentos, riscos e benefícios relacionados à pesquisa, inclusive para dirimir eventuais dúvidas.

2. liberdade de retirar seu consentimento a qualquer momento e de deixar de participar do estudo, sem que isto traga prejuízo à continuidade da assistência.

3. salvaguarda da confidencialidade, sigilo e privacidade.

4. disponibilidade de assistência no HCFMUSP, por eventuais danos à saúde, decorrentes da pesquisa.

5. viabilidade de indenização por eventuais danos à saúde decorrentes da pesquisa.

V. INFORMAÇÕES DE NOMES, ENDEREÇOS E TELEFONES DOS RESPONSÁVEIS PELO ACOMPANHAMENTO DA PESQUISA, PARA CONTATO EM CASO DE INTERCORRÊNCIAS CLÍNICAS E REAÇÕES ADVERSAS. 


\section{OBSERVAÇÕES COMPLEMENTARES:}

\section{VII - CONSENTIMENTO PÓS-ESCLARECIDO}

Declaro que, após convenientemente esclarecido pelo pesquisador e ter entendido o que me foi explicado, consinto em participar do presente Protocolo de Pesquisa
São Paulo,
de
de 20

assinatura do sujeito da pesquisa ou responsável legal

assinatura do pesquisador

(carimbo ou nome

Legível) 


\section{ANEXO II}

\section{Ficha de Avaliação}

\begin{tabular}{|c|c|c|c|c|}
\hline \multicolumn{4}{|l|}{ Nome: } & Idade: \\
\hline \multicolumn{2}{|l|}{ DN: $/ /$} & Peso: & \multicolumn{2}{|l|}{ Altura: } \\
\hline \multicolumn{2}{|l|}{$\operatorname{DMD}(\quad)$} & \multicolumn{2}{|l|}{ Normais ( ) } & \\
\hline & \multicolumn{2}{|l|}{ Medida } & \multicolumn{2}{|l|}{ Medida } \\
\hline Cirtometria & Inicial/final & & Inicial/final & \\
\hline 1 Medida & Livre & Delta & Dirigida & Delta \\
\hline \multicolumn{5}{|l|}{ Axilar } \\
\hline \multicolumn{5}{|l|}{ Xifoide } \\
\hline 2 Medida & Livre & & Dirigida & \\
\hline \multicolumn{5}{|l|}{ Axilar } \\
\hline \multicolumn{5}{|l|}{ Xifoide } \\
\hline 3 Medida & Livre & & Dirigida & \\
\hline \multicolumn{5}{|l|}{ Axilar } \\
\hline \multicolumn{5}{|l|}{ Xifoide } \\
\hline Avaliador: & & & & \\
\hline & Data: / / & & & \\
\hline
\end{tabular}




\section{ANEXO III}

\section{FICHA PARA AVALIACC̃̃O DOS INDIVIDUOS FILMADOS:}

Número:

POSTURA:

CABEÇA : Deslocamento anterior

sem deslocamento

Flexão

deslocamento posterior $\square$

Extensão

deslocamento vertical $\square$

OMBRO : Anteriorização

elevação

Posteriorização

depressão

Rotação interna

rotação externa

Sem movimentação

TRONCO: Deslocamento anterior

Deslocamento posterior

Flete

Sem movimentação

Estende 


\section{ANEXO IV}

Aprovação comissão ética

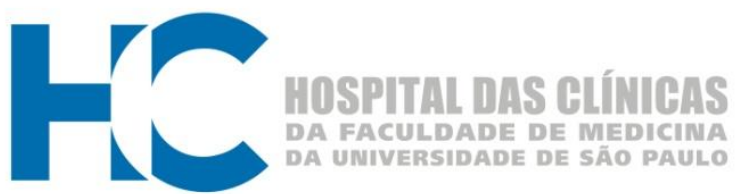

\section{APROVAÇÃo}

A Comissão de Ética para Análise de Projetos de Pesquisa - CAPPesq da Diretoria Clínica do Hospital das Clínicas e da Faculdade de Medicina da Universidade de São Paulo, em sessão de 15/07/2009, APROVOU o Protocolo de Pesquisa $\mathrm{n}^{\circ}$ 0685/09, intitulado: "CIRTOMETRIA TORÁCICA EM CRIANÇAS COM DISTROFIA MUSCULAR DE DUCHENNE - REFINAMENTO DE METODOLOGIA DE AVALIAÇÃO" apresentado pelo Departamento de FISIOTERAPIA, FONOAUDIOLOGIA E TERAPIA OCUPACIONAL, inclusive o Termo de Consentimento Livre e Esclarecido.

Cabe ao pesquisador elaborar e apresentar à CAPPesq, os relatórios parciais e final sobre a pesquisa (Resolução do Conselho Nacional de Saúde nº 196, de 10/10/1996, inciso IX.2, letra "c").

Pesquisador (a) Responsável: Fátima Aparecida Caromano

Pesquisador (a) Executante: Agenor Garcia Junior

CAPPesq, 16 de Julho de 2009



PROF. DR. CLAUDIO LEONE

Vice - Presidente da Comissão de Ética para Análise de Projetos de Pesquisa 
Referências

Capítulo: Distrofia muscular de Duchenne - função respiratória

1. HARRINSON, J. Medicina Interna. Rio de Janeiro, Editora Guanabara,11 ed, v.2, p.87-88, 1988.

2. ZATZ, M. Relação da Estatura e Peso com o Desenvolvimento Muscular e os Níveis Enzimáticos na Distrofia Muscular de Duchenne. Tese de Livre Docência apresentada ao Departamento de Biologia do IB da USP, São Paulo, 1987.

3. BARRET,R.; SCOTT. O. M. and DUBOWITS,V. Changes in Center of Gravity in boys with DMD, Muscle Nerve, vol.11, p.1157-1163. 1988

4. BAYLEY, N. The development of motor habilities during the first three years. Monogr. Soc. Res. Child. Dev., v.1, p.1-26, 1935

5. CLAUSEN, J. (Ed.) Pulmonary Function Testing Guidelines and Controversis. New York: Academic Press, 1982.

6. WEST, JOHN B. Fisiopatologia Pulmonar Moderna. Editora Manole LTDA $-4^{\circ}$ edição, 1996

7. NONAKA,L., CAROMANO,F.A,MENDES,F.AS. Avaliação de dois testes de flexibilidades em idosos do sexo feminino - método fotográfico. Rev.Ter. Ocup. Univ. São Paulo, v.10, n.2/3,p. 75-80, ma./dez., 1999.

8. CAROMANO, F. A, Características do portador de distrofia muscular de DuchenneRevisão. Arq. Ciênc. Saúde Unipar, 3(3) : 211-218, 1999.

9. GODOY, Patrìcia; NITSUMA, Lucia Emiko M.; CAROMANO, Fatima Aparecida. Avaliação funcional fisioterapêutica do respirador bucal. Arq. Ciênc. Saúde Unipar, 4(2): 111-120, 2000. 
10. GULHAM, E. G. Thoracic kyphosis, Rib Mobility, and Lung Volumes in Normal Women and Women With Osteoporosis. SPINE Volume 19, Number 11, pp 1250$1255,1994$.

11. VIGNOS, P.J.; WATKINS, M. The effect of exercise in Muscular Dystrophy, JAMA v.197, p.843-848, 1966

12. BOBATH,B.; BOBATH, K.Cerebral palsy. In Pearson P.H.: Willians C.E. (eds). Physical Services in The Developmental Disabilities, p.31-177, 1972.

13. COHEN, C. A; ZAGELBAUN,G. E GROSS, D. Clinical Manifestation of Respiratory Muscle Fatigue. Am. J. Medic. 73:308-316, 1982.

14. CHARRIÈRE, L.; ROY, J. Fisioterapia dos Desvios Laterais da Coluna Vertebral. Ed Roca, 3º edição 1987.

15. STAPE, A.; TROSTER, E.J.; KIMURA H.M.; GILIO, A.E.; BOUSSO A.; BRITTO, J.L.B.C. Manual de Normas de Terapia Intensiva Pediátrica . Ed Savier $1^{\circ}$ edição, 1998.

16. CARVALHO, C.R.R. - Series Clinicas Brasileiras de Medicina Intensiva Ventilação Mecânica Vol. I e II , 2000.

17. ROBIN, G.C.; BRIEF, L.P. Scoliosis in Chidhood Muscular Dystrophy.J.Bone and Joint Surg. V.53. A, n.4, p. 466-476, 1971.

18. BOUISSET S, DUCHENNE JL (1994) Is body balance more perturbed by respiration in seatingposture Neuroreport 5: 957-960

19. GURFINKEL VS, KOTS YM, Paltsev El, Feldman AG (1971) The compensation of respiratory disturbances of erct posture of man as an example of the organization of interaction. In Gelfand IM, Gurfinkel VS, Formin SV, Tsetlin ML (eds) Models of the structural functional organization of certain biological systems. MIT Press, Cambridge, MA, pp 382-395. 
20. HODGES PW, CRESSWELL AG, TRORSTENSSOM A (1999) Preparatory trunk motion accompanies rapid upper limb movement. Exp Brain Res 124:69-79

21. HUNTER IW, KEARNEY RE (1981) Respiratory components of human postural sway. Neurosci Lett 25: 155-159.

22. WALTON J. GARDNER-MEDWIN D. - The muscular dystrophies. In: Walton J. Disorders of Voluntary muscle. London, Churchilt Livingstone, 5 th ed., Cap 15,pp 519-569, 1988.

23. BUREAU MA, BEGIN R. - Chest Wall Diseases and Dysfunction in Children In Kendig EL. Chermick V. - Disorders of the Respiratory Tract in Children Philadelphia WB Saunders Co. 4a. ed. Cap44, pp 609-614.

24. HECKMATT JZ - Respiratory care in muscular dystrophy. Br Med J, 295: 10141015,1987

25. VERENUSO G. ADAMILANI C, AZZARI C, CALANDI C, FRANCHINI C, SABATINI C, VIERUCCIA - Evaluation of the respiratory function in pacients with Duchenne's muscular dystrophy. Pediatr med Chir, 9(1): 15-19, 1987

26. SMITH AD, KORESKA J, MOSELLEY CF - Progression of scoliosis in Duchenne muscular dystrophy. J Bone Joint Surg, 71 (7) 1066-1074, 1989.

27. Silva EF, Guedes RP, Ribeiro EC. Estudo das repercussões das cirurgias abdominais -sobre os músculos respiratórios. Fisioter Mov 2003;16(1):51-6.

28. Pires AC, Saporito WF, Grandini M, Oliveira AC, Zambon JP, Ávila MP. Avaliação da disfunção diafragmática no pós-operatório de cirurgia cardíaca. Arq Med ABC $1999 ; 22(1 / 2): 13-7$

29. Garcia RCP, Costa D. Treinamento muscular respiratório em pós-operatório de cirurgia cardíaca eletiva. Rev Bras Fisioter 2002;6(3):139-46. 
30. Maciel SS, Paulo MQ, Souza CO, Silva LG, Tavares RR. Efeito broncodilatador do Acanthosperum hispidum DC, nos pacientes pulmonares obstrutivos crônicos (DPOC). Rev Bras Cienc Saúde 1997;1(1/3):23-30.

31. Aliverti A, Stevenson N, Dellaca RL, Lo Mauro A, Pedotti A, Calverley PM. Regional chest wall volumes during exercise in chronic obstructive pulmonary disease. Thorax 2004;59(3):210-6.

32. Ringel ER, Loring SH, McFadden ER Jr, Ingram RH Jr. Chest wall configurational changes before and during acute obstructive episodes in asthma. Am Rev Respir Dis 1983;128(4):607-10.

33. Caromano FA, Durigon OFS, Landaburu C, Pardo MS. Estudo comparativo de duas técnicas de avaliação da mobilidade torácica em mulheres jovens e idosas saudáveis. Fisioter Bras 2003;4(5):348-52.

34. Costa D, Sampaio LMM, Pires Di Lorenzo VA, Jamami M, Damaso AR. Avaliação da força muscular respiratória e amplitudes torácicas e abdominais após RFR em indivíduos obesos. Rev Latinoam Enfermagem 2003;11(6):156-60.

35. De Groote A, Wantier M, Cheron G, Estenne M, Paiva M. Chest wall motion during tidal breathing. J Appl Physiol 1997;83(5):1531-7.

36. Manczur T, Greenough A, Hooper R, Allen K, Latham S, Price JF, et al. Tidal breathing parameters in young children: comparison of measurement by respiratory inductance plethysmography to a facemask pneumotachograph system. Pediatr Pulmonol 1999;28(6):436-41.

37. Martucci RC, Lopes JM, Jardim JRB. Chest wall configuration in normal subjects in the sit and supine positions breathing free and though a linear resistence. J Pneumol 1992;18(3):93-100. 
38. Jamami M, Pires VA, Oishi J, Costa D. Efeitos da intervenção fisioterápica na reabilitação pulmonar de pacientes com doença pulmonar obstrutiva crônica (DPOC). Rev Fisioter Univ São Paulo 1999;6(2):140-53.

39. Salomão PDN, Silva AL. Repercussões respiratórias funcionais da hernioplastia incisional abdominal longitudinal. Rev Col Bras Cir 1995;22(1):33-41.

40. Mcardle WD, Katch FI., Katch VL. Fisiologia do exercício. 4a ed. Rio de Janeiro: Guanabara Koogan; 1998.

41. Sampaio LMM. Adaptações fisiológicas do paciente asmático ao exercício físico [dissertação]. São Carlos: Universidade Federal de São Carlos; 2002.

42. Silva AB. Efeitos da suplementação de L-carnitina em pacientes com DPOC submetidos a um programa de reabilitação pulmonar [dissertação]. São Carlos: Universidade Federal de São Carlos; 2002.154p.

43. Cardoso RX, Pereira JS. Análise da função respiratória na doença de Parkinson. Arq Neuropsiquiatr 2002;60(1):91-5.

44. Naimark A, Chemiak RM. Compliance of the respiratory system and its components health and obesity. J Appl Physiol. 1960; 15: 377-82

45. Wipp BJ, Wasserman R. Exercise,I.: Murray JF, Nadel JA, editoris. Textbook of respiratory medicine. $2^{\text {nd }}$ ed . Philadelphia: WB, Saunders; 1996 p.246.

46. Gilroy RJ, Mangura BT, Lavietes MH. Rib cage and abdominal volume displacements during breathing in pregmancy. Am Rev Respir Dis. 1988; 137: 66872.

47. Sharp JT, Henry JP, Sweany SR, Meadwos WR, Pietras RJ. The total work of breathing in normal and obese men. J Clin Invest. 1964;43: 728-39.

48. Zambom L.,Saad IAB. Variáveis Clinicas de Risco Pré-operatório. Assoc. Méd. Brás. 2001; 47 : 227-3 
49. Chiavegato LD, Jardim JR. Alterações funcionais respiratórias colecistectomia por via laparoscópica. J. Pneumologia 2000; 26,2: 1-15.

50. Galdiner BN, Leary BF, Wolfe BM. Endoscopic cholecystectomy: an analysis of complication. Arch. Surg. 1996;12: 46-52.

51. CRAIG DB. Potoperative recovery of pulmonary function. Anesth Analg. 1994;60: $46-52$.

52. Celli B. Perroperative Respiratory care of the patient undergoing upper abdominal surgery. Chest 1993, 14: 253-61.

53. Jamami M, Pires VA, Oishi J, Costa D. Efeitos da intervenção fisioterápica na reabilitação pulmonar de pacientes com doença pulmonar obstrutiva crônica (DPOC). Rev Fisioter Univ São Paulo 1999;6(2):140-53.

54. G. FONSECA et al. Distrofia muscular de Duchenne:complicações respiratórias e seu tratamento Rev. Ciênc. Méd., Campinas, 16(2):109-120, mar/abr., 2007

55. Kennedy JD, Staples AJ, Brook PD. Effect of spinal surgery on lung funtion in Duchenne muscular dystrophy. Thorax. 1995; 50:1173-8.

56. Koessler W, Wanke T, Winkler G, Nader A, Toifl K, Kurz H, et al. 2 years'

57. Experience with inspiratory muscle training in patients with neuromuscular disorders. Chest. 2001; 120(3):765-9.

58. Szeinberg A, Tabachnik E, Rashed N, McLaughlin FJ, England S, Bryan CA, et al. Cough capacity in patients with muscular dystrophy. Br J Dis Chest.1988;94:12325 .

59. Hopke EJ, Meek JC, Jacobs J. Pulmonary function in progressive muscular dystrophy. Br J Dis Chest.1972;61(1):41-7. 


\section{Capítulo: Postura Sentada e Função Respiratória}

1. American Thoracic Society. Lung function testing: selection of reference values and interpretative strategies. Am Rev Respir Dis 1991;144:1202-18. J Pneumol 28(Supl 3) - outubro de 2002 S 93

2. Quanjer PH, Tammeling GJ, Cotes JE, Pedersen OF, Peslin R, Yernault JC. Lung volumes and forced ventilatory flows. Report of Working Party. "Standardization of Lung Function Tests", European Community for Steel and Coal and European Respiratory Society. Eur Respir J 1993;16(Suppl):5-40.

3. Comroe JH Jr, Forster RE II, DuBois AB, Briscoe WA, Carlsen E. The lung: clinical, physiology and pulmonary function tests. 2nd ed. Chicago: Year Book Medical Publishers Inc., 1962.

4. Gibson GJ. Lung volumes and elasticity. In: Hughes JMB, Pride NB. Lung function tests. Physiological principles and clinical applications. London: WB Saunders, $1999 ; 45-56$.

5. Vincken W, Ghezzo H, Cosio MG. Maximal static respiratory pressures in adults: normal values and their relationship to determinants of respiratory function. Bull Eur Physiopathol Respir 1987;23:435-9.

6. Bruschi C, Cerveri I, Zoia MC, Fanfulla F, Fiorentini M, Casali L, et al. Reference values of maximal respiratory mouth pressures: a population-based study. Am Rev Respir Dis 1992;146:790-3.

7. Rubinstein I, Slutsky AS, Rebuck AS, McClean PA, Boucher R, Szeinberg A, et al. Assessment of maximal expiratory pressure in healthy adults. J Appl Physiol 1988;64:2215-9. 
8. Koulouris N, Mulvey DA, Laroche CM, Green M, Moxham J. Comparison of two different mouthpieces for the measurements of PImax and PEmax in normal and weak subjects. Eur Respir J 1988;1:863-7.

9. Wanger J. Pulmonary function testing. A practical approach. 2nd ed. Baltimore: Williams \& Wilkins, 1996.

10. Leff AR, Schumacker PT. Respiratory physiology: basics applications. Philadelphia: WB Saunders Co., 1993.

11. OKOSHI, M.P.; CAMPANA, A.O.; GODOY, I. Exame Físico do Tórax - Aparelho Respiratório. A Revista da Clínica Médica. V.30, n.9, p.33-53, 1997.

12. AZEREDO, C.A.C. Fisioterapia respiratória. São Paulo: Manole, 1984.

13. CARVALHO, A. Semiologia em reabilitação. São Paulo: Atheneu, 1994.

14. BETHLEM, N. Pneumologia. 4 ed. São Paulo: Atheneu, 1995.

15. LIANZA, S. Medicina de reabilitação. 2 ed. Rio de Janeiro: Guanabara Koogan, 1995.

16. Carvalho MRA. Avaliação morfodinâmica do tórax e do abdomen. In: Carvalho MRA, editor. Fisioterapia respiratória: fundamentos e contribuições. Rio de Janeiro: Nova Casuística; 1979. p. 65-68.

17. Cardoso SRX, Pereira JS. Análise da função respiratória na doença de Parkinson. Arq Neuropsiquiatr. 2002;60(1):91-5.

18. Maciel SS, Paulo MQ, Souza CO, Silva LG, Tavares RR. Efeito broncodilatador do Acanthospermum hispidum DC, nos doentes pulmonares obstrutivos crônicos (DPOC). Rev Bras Cienc Saúde. 1997;1(1/3):23-30.

19. Anderson JM. Assessment of chest function by the physiotherapist. In: Cash JE, Downie PA, editors. Cash's Textbook of chest, heart, and vascular disorders for physiotherapists. Philadelphia: Lippincott; 1987. p. 318-24. 
20. Kakizaki F, Shibuya M, Yamazaki T, Yamada M, Suzuki H, Homma I. Preliminary report on the effects of respiratory muscle stretch gymnastics on chest wall mobility in patients with chronic obstructive pulmonary disease. Respir Care. 1999;44(4):409-14.

Capítulo: Toracometria em crianças com distrofia muscular de Duchenne - expansão de metodologia de avaliação

1. López-Hernández LB, Vázquez-Cárdenas NA, Luna-Padrón E. Duchenne muscular dystrophy: current aspects and perspectives on treatment. Rev Neurol. 2009 Oct 115;49(7):369-75.

2. Allen J. Pulmonary complications of neuromuscular disease: a respiratory mechanics perspective. Paediatr Respir Rev. 2010 Mar;11(1):18-23.

3. Smith AD, Koreska J, Moselley CF. Progression of scoliosis in Duchenne muscular dystrophy. J Bone Joint Surg, 71 (7) 1066-1074, 1989.

4. Verenuso G. Adamilani C, Azzari C, Calandi C, Franchini C, Sabatini C, Vieruccia-Evaluation of the respiratory function in pacients with Duchenne's muscular dystrophy. Pediatr Med Chir, 9(1): 15-19, 1987.

5. Inkley SR, Oldenburg FC, Vignos PJ. Pulmonary function in Duchenne muscular dystrophy related to stage of disease. Am J Med. 1974;56:297-306.

6. Estenne A, Heilporn A, Delhez L, Yernalt JC, Troyer A. Chest wall stiffness in patients with chronic 
respiratory muscle weakness. Am Rev Respir Dis. $1983 ; 128: 1002-1007$.

7. L.D. Joanne, R.P. Woda and J.R. Mendell, Respiratory dysfunction in muscular dystrophy and other myopathies. Clin Chest Med 15. 1994; 661-674.

8. Bushby K, Finkel R, Birnkrant DJ, Case LE, Clemens PR, Cripe L, Kaul A, Kinnett K, mcdonald C, Pandya S, Poysky J, Shapiro F, Tomezsko J, Constantin C..Diagnosis and management of Duchenne muscular dystrophy, part 2: implementation of multidisciplinary care. Lancet Neurol. 2010 Feb;9(2):177-89.

9. Costa D, Sampaio LMM, Pires Di Lorenzo VA, Jamami M, Damaso AR. Avaliação da força muscular respiratória e amplitudes torácicas e abdominais após RFR em indivíduos obesos. Rev Latinoam Enfermagem 2003;11(6):156-60.

10. Bockenhauer SE, Chen H, Julliard NK, Weedon J. Measuring Thoracic Excursion: Reliability of the Cloth Tape Measure Technique. J Am Osteopath Assoc. 2007;107:191-196.

11. Eadie, W.T.; D. Drijard, F.E. James, M. Roos and B. Sadoulet (1971). Statistical Methods in Experimental Physics. Amsterdam: North-Holland. pp. 269-271.

12. Siegel, S.; Castellan, N,J, Estatística Não-paramétrica para Ciência do Comportamento. Editora Artemed, SP, 2a Ed. 2006.

13. Weir, JP. Quantifying test-retest reliability using the intraclass correlation coefficient and the sem. Journal of Strength and Conditioning Research. 2005; 19(1): 231-240.

14. Iunes DH et al. Confiabilidade intra e interexaminadores e repetibilidade da avaliação postural pela fotogrametria. Revista Brasileira de Fisioterapia. 2005; 9 (3): 327-34.

15. Levin, Jack; Fox, James Alan Estatística para Ciências Humanas. 9ª Ed. 2004. p 497.

16. Landis JR, Koch GG. The measurement of observer agreement for categorical data. Biometrics.1977; 33:159-75.

17. Silva EF, Guedes RP, Ribeiro EC. Estudo das repercussões das cirurgias abdominais sobre os músculos respiratórios. Fisioter Mov 2003;16(1):51-6. 
18. Pires AC, Saporito WF, Grandini M, Oliveira AC, Zambon JP, Ávila MP. Avaliação da disfunção diafragmática no pós-operatório de cirurgia cardíaca. Arq Med ABC 1999;22(1/2):137.

19. liverti A, Stevenson N, Dellaca RL, Lo Mauro A, Pedotti A, Calverley PM. Regional chest wall volumes during exercise in chronic obstructive pulmonary disease. Thorax 2004;59(3):210-6.

20. Bockenhauer SE, Julliard KN, Lo KS, Huang E, Sheth AM. Quantifiable effects of osteopathic manipulative techniques on patients with chronic asthma. J Am Osteopath Assoc. 2002;102:371375.

21. Zambom L.,Saad IAB. Variáveis Clinicas de Risco Pré-operatório. Assoc. Méd. Brás. 2001; 47:227-3

22. Custers JWH, Arets HGM, Engelbert RHH, Kooijmans FTC. Thoracic excursion measurement in children with cystic fibrosis. Journal of Cystic Fibrosis 4. 2005; 129 - 133

23. C.S.B. Galasko J. B. Willianson, and C.M. Delaney: Deterioration of lung function and scoliosis in Duchenne muscular dystrophy. Eur Spine J (1995) 4: 263-267.

24. Heller KD, Forst R, Forst J, Hengstler K. Scoliosis in Duchenne muscular dystrophy: aspects of orthotic treatment. Prosthet Orthot Int. 1997 Dec;21(3):202-9.

25. Yamashita T, Kanaya K, Yokogushi K, Ishikawa Y, Minami R. Correlation between progression of spinal deformity and pulmonary function in Duchenne muscular dystrophy. J Pediatr Orthop. 2001 Jan-Feb;21(1):113-6.

26. Jover M, Schmitz C, Bosdure E, Chabrol B, Assaiante C. Anticipatory postural adjustments in a bimanual load-lifting task in children with Duchenne muscular dystrophy. Neuroscience Letters 403. 2006; 271-275.

27. Kang SW, Kang SY, Moon HJ, Yoo WT. Assisted Cough and Pulmonary Compliance in Patients with Duchenne Muscular Dystrophy. Yonsei Medical Journal 2005; 46: 233 - 238.

28. Park JH, Kang SW, Lee SC, Choi WA, Kim DH. How Respiratory Muscle Strength Correlates with Cough Capacity in Patients with Respiratory Muscle Weakness. Yonsei Med Journal 2010; 51(3): 392-397.

29. Mauro LA, et al. Abdominal volume contribution to tidal volume as an early indicator of respiratory impairment in Duchenne muscular dystrophy. Eur Respir J 2010; 35: 1118-1125. 
30. Gulham, E. G. Thoracic kyphoss, Rib Mobility, and Lung Volumes in Normal Women and Women With Osteoporosis. SPINE 1994; 19(11): 1250-1255. 